\title{
Exploring Research in Blockchain for Healthcare and a Roadmap for the Future
}

\author{
Mohamad Kassab, Member, IEEE, Joanna DeFranco, Senior Member, IEEE, \\ Tarek Malas, Phillip Laplante, Fellow, IEEE, Giuseppe Destefanis, and Valdemar Vicente Graciano Neto
}

\begin{abstract}
Healthcare is a data-intensive domain, once a considerable volume of data is daily to monitoring patients, managing clinical research, producing medical records, and processing medical insurance claims. While the focus of applications of blockchain in practice has been to build distributed ledgers involving virtual tokens, the impetus of this emerging technology has now extended to the medical domain. With the increased popularity, it is crucial to study how this technology accompanied with a system for smart contracts can support and challenge the healthcare domain for all interrelated actors (patients, physicians, insurance companies, regulators) and involved assets (e.g. patients' data, physician's data, equipment's and drug's supply chain, etc.). The contributions of this paper are the following: (i) report the results of a systematic literature review conducted to identify, extract, evaluate and synthesize the studies on the symbiosis of blockchain in healthcare; (ii) summarize and categorize existing benefits/challenges on incorporating blockchain in healthcare domain; (iii) provide a framework that will facilitate new research activities; and (iv) establish the state of evidence with in-depth assessment.
\end{abstract}

Index Terms—Blockchain, Healthcare, Medicine, Hyperledger.

\section{INTRODUCTION}

$\mathrm{H}$ EALTHCARE is a data-intensive discipline [1] in which largescale data is generated, disseminated, stored, and accessed daily. In 2017, 16.5 million patients globally exploited remote health monitoring (a $41 \%$ growth from 2016), and this panorama has the potential to reach 50.2 million by 2021 [2]. Also, since January 1st, 2018, the U.S. Centers for Medicare \& Medicaid Services ${ }^{1}$ developed new reimbursement incentives to promote the adoption of "active feedback loop" devices to provide real-time monitoring [3]. Data created when a patient is monitored or undergoes some tests, need to be stored in order to be accessible at a later time by a healthcare provider within the same or even a different network or context. As this realm expands, concerns about secure and efficient transmission of the medical data increase. Despite these concerns, polls show that $90 \%$ of Americans still value online access to their health records [4]. It is easy to perceive that technology can contribute to enhancing the quality of caregiving for patients at a reduced cost. For example, in 2014, 15\% of U.S. patients who visited a healthcare provider reported having to bring their medical test results to their appointment, and $5 \%$ required to have a procedure or a test replicated due to the lack of access to a prior test result [5].

Blockchain technology has enjoyed substantial deliberation in recent years from the financial/banking disciplines. The technology of blockchain attracted considerable attention due to the possibility of recording all financial transactions in a secure and verifiable decentralized (peer-to-peer) fashion, without the rule from a third party to process transactions, which are then combined into blocks where each block contains a timestamp and is linked to its precedent. Once recorded, data cannot be altered, and the transactions history is combined into a chain structure without the possibility of additional branches of alternative transactions emerging or wedging into the middle of a chain [6].

While the focus of blockchain applications in practice has been to build distributed ledgers involving virtual tokens (e.g., cryptocur-

1. https://www.cms.gov/ rencies), this technology has recently gained increased attention in several other fields. In a January 2016 report, Mark Walport, the U.K. government's chief scientific adviser argued that blockchain technology could expand far beyond a trading tool: "Distributed ledger technologies have the potential to help governments collect taxes, deliver benefits, issue passports, record land registries, assure the supply chain of goods, and generally ensure the integrity of government records and services," the report concluded [7].

The impetus has now extended to the medical domain. Starting from the last half of 2017, we saw an uptick in interest from healthcare giants to get involved in blockchain, whether in joining consortium efforts like Hyperledger ${ }^{2}$ or developing their services and products. As of the beginning of September 2017, 15 publications were found in the electronic medical library PubMed ${ }^{3}$ as a result of the search query "blockchain" [6]. A similar search we conducted in December 2018 yielded 98 publications. The textual analysis of these publications highlights the potential of utilizing the blockchain to improve transparency and sharing of health records, improve the quality of clinical trials and prevention of distortion of scientific conclusions, and to contribute to managing the drugs supply chain effectively.

There are both advantages and disadvantages to centralized (traditional database management systems) vs decentralized data architectures. It is also not an either/or decision - the opportunity before the medical sector is with developing hybrid architectures that appropriately leverage the strengths of each set of technologies for the uses they are best suited for.

The contributions of this paper are the following: (i) report the results of a systematic literature review (SLR) conducted to identify, extract, evaluate and synthesize the studies on the symbiosis of blockchain in healthcare [8]; (ii) summarize and categorize existing benefits/challenges on incorporating blockchain in healthcare domain; (iii) provide a framework that will facilitate new research

2. https://www.hyperledger.org/

3. https://www.ncbi.nlm.nih.gov/pubmed/ 
activities; and (iv) establish the state of evidence with in-depth assessment.

While we presented an initial view from the review in short papers [9], [10] focusing only on the usage of blockchain in managing the healthcare records, this paper presents the extended results from the review along with the complete set of mappings and process descriptions.

The remainder of this paper is structured as follows. Section 2 provides a background on blockchain. Section 3 presents our research method, research questions and a high-level overview of the selected studies. Section 4 summarizes our findings on the extracted scenarios. Section 5 discusses the keys benefits of incorporating blockchain for healthcare, while Section 6 discusses the challenges. Section 7 brings a more in-depth discussion based on the results and draws a road map for future research, while Section 8 concludes the paper and discusses the limitations of this study.

\section{BACKGROUND}

The theory behind Bitcoin as a peer-to-peer electronic cash system was introduced in a white paper written under the pseudonym "Satoshi Nakamoto" in 2008 [11]. A decade later, and despite the uncertainty of the identity of its creator, Bitcoin was rapidly implemented and widely accepted as a prominent online cryptocurrency. This is evidenced by the total USD value of Bitcoin supply in circulation at the time of writing this paper which hovers around $\$ 100$ billion dollars (as of May 2019) ${ }^{4}$. Many online retailers accept Bitcoin as a mean of payment with many mechanisms in existence for exchanging it with fiat currency and vice versa [12].

The blockchain is the essence of the infrastructure underlying Bitcoin and other cryptocurrencies. In practice a blockchain is built upon a chronological chain of block-like data structures, hence its name. A block hosts with a timestamped set of transactions that are bundled together. Each new block is linked to its preceding block. Combined with cryptographic hashes, this time-stamped chain of blocks provides a hopefully "immutable" record of all transactions in a network, from the genesis block until the last / most current block. Figure 1 depicts a standard structure for a blockchain, which consists of the following four pieces of metadata:

1) previous block reference

2) proof of work (a.k.a. a nonce)

3) time-stamp

4) Merkle tree root for the block transactions

A blockchain comprises a set of nodes without a preexisting trust relationship and connected through a peer-to-peer topology. Each node hosts the same copy of a blockchain creating a decentralized structure. However, for such a structure to be useful, there must exist some mechanism by which the nodes can mutually reach a consensus on the next valid block in the chain to be added. The consensus mechanisms are protocols that make sure all nodes (devices that maintain the blockchain and, sometimes, process transactions) are synchronized with each other and agree on which transactions are legitimate and are added to the blockchain. These consensus mechanisms are crucial for the precise function of a blockchain. Some of the schemes adopted for establishing a distributed consensus include Proof of Work, Proof

4. As of 04-May-2019: There are 17.7 million bitcoins in circulation. The exchange value for the U.S. Dollar is $5660 \$$. https://charts.bitcoin.com/btc/ of Stake, Proof of Capacity, Proof of Human-Work, Proof of Activity and Proof of Elapsed Time [13], [14], [15].

A blockchain is an append-only distributed ledger. In other words, the new entries get added at the end of the ledger. In contrast with a traditional relational database where data can be deleted or altered, there are no administrator's permissions within a blockchain that allow for deleting or editing of the recorded data. Furthermore, unlike a centralized relational database, blockchains are designed for decentralized applications. This immutability feature implies that once a transaction is added onto the blockchain, no one can alter it. This makes blockchain an ideal solution for assets transactions and identity management, to mention a few examples.

In addition to decentralization, consensus and immutability, a blockchain network has two additional key characteristics: Provenance and finality. Provenance refers to awareness that participants of the network have about where the asset was originated from and its ownership history, while finality refers to the status of a transaction as complete.

A blockchain can use smart contracts, which are stored on the blockchain and executed automatically to serve as agreements or a set of rules that oversee a blockchain transaction. For example, a smart contract may define the contractual conditions of an individual's travel insurance. The conditions will automatically execute upon notice of a flight delay by more than a certain number of hours [14].

A blockchain can be both permissionless (public) or permissioned (private). In a permissionless blockchain, any node can join the network. In a permissioned (private) blockchain, preverification of the participating parties, which are all known to each other, is required. The choice between the two types is mainly driven by the use case in a particular application. If a network can 'commoditize' trust, where the identity of the facilitating parties does not need to be verified, a permissionless blockchain makes sense. An example of a permissionless blockchain is Bitcoin or Ethereum. On the other hand, managing the medical healthcare records is an ideal use case for a permissioned blockchain as it makes sense to have the participating companies vetted. In other words, when it is vital that the blockchain participants require permission to execute transactions, a permissioned blockchain makes sense. This also helps all participants in the network to understand where the transactions are originated from. Hyperledger - an opensource blockchain initiative hosted by the Linux Foundation - is an example of a permissioned blockchain.

The success of the Bitcoin currency system has paved the way for similar ledger systems that are decentralized and open. blockchain can be a crucial technology to transaction-based information, similar to the way networking protocols allowed for the growth of the Internet and current media streaming services. Within a few years, it is estimated that blockchain will have enabled savings of $\$ 15$ to $\$ 20$ billion annually [16].

In Melanie Swan's book "Blockchain, Blueprint for a New Economy" [15], blockchain adoption was predicted in three phases. The current phase, Blockchain 1.0, is defined as the online cryptocurrency phase (e.g. Bitcoin). Blockchain 2.0, occurring in the near future, expand phase 1.0 to "encompass tracking contracts, financial records, public records, and ownership of property. Examples of Blockchain 2.0 systems could include fraud- and errorresistant land ownership records databases". Moreover, finally, her vision for Blockchain 3.0 would expand into "science, medicine, and education. She predicts information that has been hidden 


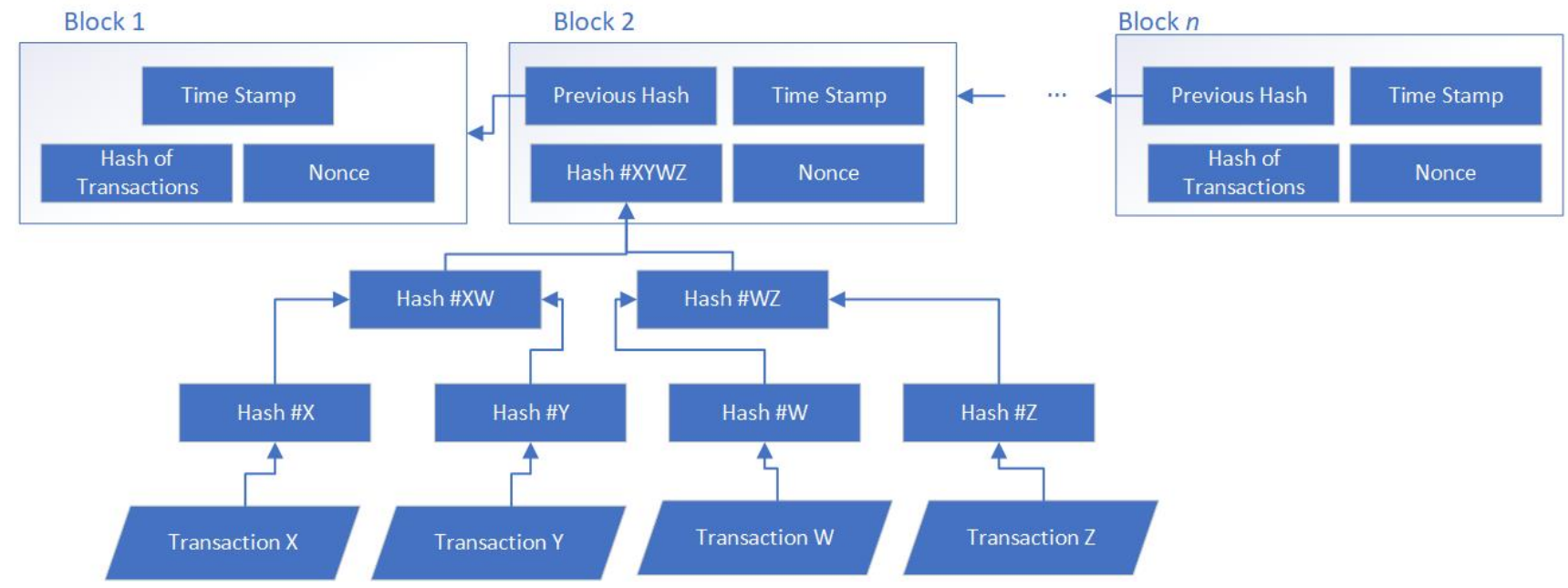

Fig. 1. A common structure of a Blockchain

and controlled within institutions will be moved to open and distributed blockchains". These possible uses for Blockchain 3.0 should be the most intriguing to readers of this paper as we explain in the next sections.

\section{Systematic MAPPING ON BLOCKChain FOR HEALTHCARE}

In this research, we followed the guidelines and SLR protocol template proposed by Kitchenham and Charters [8]. A software tool was used to support the SLR protocol definition and conducting the review process. The tool, called StArt (State of the Art through Systematic Review) ${ }^{5}$, has been empirically evaluated, and it was demonstrated having positive results in the execution of SLRs [17].

\subsection{Planning The Review}

We planned the review process by refining the research objectives into a set of research questions. We also identified the search strategy, search strings, inclusion/exclusion criteria and the quality assessment criteria to be applied to the extracted studies.

\subsubsection{Review Objectives and Research Questions}

With the increased popularity of blockchain in the healthcare domain, it is crucial to study how this technology can support and challenge the healthcare domain for all interrelated actors (patients, physicians, insurance companies, regulators) and involved assets (e.g. patients' data, physician's data, equipment's and drug's supply chain, etc.). One of the goals of this work is to develop an understanding of the scenarios that involve deploying blockchain in healthcare, the benefits that arise from this incorporation and the challenges in such a context. To fulfil the objectives, we formulated three main research questions:

- RQ1. What are the present scenarios in discussion for the potential usages of blockchain in healthcare?

- RQ2. What are the benefits of the adopted scenarios of blockchain in healthcare?

- RQ3. What are the challenges of incorporating blockchain in healthcare?

5. http://lapes.dc.ufscar.br/tools/start_tool
In order to facilitate answering the first question, six subresearch questions emerged:

- RQ1.1 Who are the actors involved in a discussed scenario?

- RQ1.2 When applicable, what is the medical condition (e.g., disease) which is addressed by a scenario?

- RQ1.3 Which healthcare transactions are intended to be stored within a distributed ledger according to a scenario?

- RQ1.4 Which areas of healthcare are addressed through an emerging scenario (e.g. monitoring, diagnosis, treatment, insurance, education, etc.)?

- RQ1.5 In which depth a scenario was further leveraged in practice (e.g., designed, implemented, deployed, verified)?

- RQ1.6 Was a particular blockchain technology (e.g., Fabric) utilized for a discussed scenario?

\subsubsection{Search Strategy}

Following the study by Kitchenham and Charters [8] as a guideline for carrying out the research, we defined the search space which utilized PennState LionSearch tool to look for manuscripts. LionSearch is an integrated search engine of books, e-books, research articles, newspaper articles, and other publications integrated from over 950 database/search engines, including over 80 databases/search engines for healthcare/medicine discipline and over 15 for computer/software/information science and engineering. The tool is provided and maintained by the Pennsylvania State University's Library.

In order to obtain a comprehensive view when answering our questions, it was essential to run our search on specialized domains from the two worlds: the computer science/ engineering discipline and the healthcare discipline, hence our selection for five domains to filter our search in LionSearch: "Computer Science", "Medicine", "Dentistry", "Nursing" and "Pharmacy, Therapeutics Pharmacology". The search was conducted in "December 2018" with no timelines applied for the publication period (Table 1).

The initially retrieved studies from the electronic databases were assessed. Then, the inclusion and exclusion criteria, explained below, were applied to all of the studies kept after an initial assessment to filter the remaining papers. 
TABLE 1

Search Sources.

$\begin{array}{lc}\text { Electronic Databases } & \begin{array}{r}\text { Penn State LionSearch engine (integrating over } \\ 950 \text { databases / search engines) }\end{array} \\ \text { Searched Items } & \begin{array}{c}\text { Journal, workshop, magazine and conference } \\ \text { papers (peer reviewed items) }\end{array} \\ \text { Search applied on } & \begin{array}{c}\text { Full text - not to miss relevant } \\ \text { papers where the keywords were not } \\ \text { in the title or abstract. }\end{array} \\ \text { Language } & \text { English } \\ \text { Publication Period } & \begin{array}{c}\text { From the beginning of the } \\ \text { time till December } 2018\end{array}\end{array}$

\subsubsection{Search Criteria}

The search criteria used for this review consist of two parts defined as follows:

- C1 is a string made up of the two keywords "Blockchain" and "Hyperledger".

- C2 is a string made up of keywords related healthcare such as "Medicine", "Healthcare" and "Nursing".

The Boolean expression search criterion was "C1 AND C2". An example of a search done in the LionSearch is: "Blockchain" OR "Hyperledger") AND ("Medicine" OR "Healthcare" OR "Nursing”).

\subsubsection{Inclusion and Exclusion Criteria}

To determine whether a study should be included, we used the following inclusion and exclusion criteria:

Inclusion criteria: (I1) the paper represents a peer-reviewed study; (I2) the language is English; (I3) it is relevant to the search terms defined; (I4) it is an empirical research paper, an experience report, a proof of concept, a visionary article or workshop paper.

Exclusion criteria: (E1) study's focus is not explicitly on blockchain; (E2) study that does not address the healthcare domain; (E3) study that does not meet all the inclusion criteria; and (E4) prefaces, keynote, viewpoint, editorial comments, tutorials, anecdote papers and only presentations slides.

\subsection{Conducting The Review}

In this section, we present the process of conducting our search and the extraction of studies and information from the mentioned databases.

\subsubsection{Study Search and Selection}

By following the search strategy, the selected search engine was queried. The search results (197 papers) were automatically downloaded, organized and entered with the aid of the StArt tool. Figure 2 depicts the seven steps of the studies' selections with the number of studies resulting in each one these steps.

At Step 2, duplicated studies were automatically identified and removed using the StArt tool, leaving a set of 173 papers. Then, in Step 3, 42 papers were removed based on the exclusion criteria E4 or when the primary language was not English. At Step 4, authors screened all the remaining 129 papers. For screening the relevant papers, we used a process inspired by Dybå and Dingsøyr [18] to first review the titles, keywords, abstracts and publication local of each paper and exclude those according to the exclusion criteria
- Identify and organize studies retrieved from LionSearch. [197 papers retrieved].

-Automatic removal of duplicated papers using StArt tool. [171 papers remained]

-Applying Exclusion Critera (E4): Excluding prefaces, keynote, viewpoint, editorial comments, tutorials, anecdote papers and only presentations slides. Excluding non-English. [129 papers remained].

- Screening all selected papers from step 3 for inclusion / exclusion criteria. [54 papers remained].

- Keywording using abstracts and establishing the classification scheme. [Applied on the $\mathbf{5 4}$ remaining papers].

the 3 are retrieved and those that attended the inclusion criteria were selected. [54 papers remained].

-The papers that did not satisfy a minumum of $50 \%$ quality score were excluded. [52 papers remained].

Fig. 2. Papers Selection Process.

(75 excluded at this step). If there was insufficient data, the paper was left for the next assessment step.

At Step 5, we conducted keywording to construct a classification scheme. We used the process defined by Petersen et al. [19]. Keywording was conducted by: 1) reading the abstract and identifying keywords and concepts that constitute the contributions of the paper [19]. 2) then developing a higher level of understanding based on these keywords [19]. The keywords helped us to cluster and form categories for the mapping of the studies. In step 6 and after the categories had been clustered, we read all the selected papers. The full text of each paper was reviewed thoroughly by at least two authors. To review the agreements raised in the assessments, we conducted several consensus meetings along the process and also updated the categories, if a study revealed something new.

In the last step, and in order to assess the methodological quality of the primary studies selected for this review, we adopted the quality criteria initially proposed in the "Users' Guides to the Medical Literature" published in [20] and presented in Table 2. The reasons that supported our choice for those criteria are: those guidelines (i) can be utilized to investigate the usefulness of synthesis findings [8] and (ii) were previously employed successfully in several recent systematic reviews (e.g., [18], [21]). We evaluated each study according to these quality assessment criteria, which is made up of questions that act as an assessment tool to determine the extent to which each study contributes to our investigation. The normalized scores of the selected studies, which are based on their quality scores, was calculated. We set a " 0.5 " quality score as the minimum score of accepting studies for this search. Two studies were excluded based on not satisfying the quality score.

\subsubsection{Data Extraction and Synthesis}

The data extraction process was conducted by reading the fulltext for each one of the 52 selected primary studies. In order to guide this data extraction, the data collection from Kitchenham and Charters [8] was adopted. In particular, we utilized a predefined extraction form (that we published at https://goo.gl/4VDp2i) to record the full details of the studies under review and to be specific about addressing our research questions. StArt tool was utilized in the extraction process as well. 
TABLE 2

QUALITY CRITERIA FOR STUDY SELECTION.

\begin{tabular}{cc} 
Criteria & Response Grading \\
\hline $\begin{array}{c}\text { (C1) Is the research aim/objective } \\
\text { clearly defined? }\end{array}$ & $\begin{array}{c}1,0.5,0 \\
\text { (Yes, nominally, No) } \\
\text { (C2) Is the context of } \\
\text { research well addressed? }\end{array}$ \\
$\begin{array}{c}1,0.5,0 \\
\text { (C3) Are the findings } \\
\text { clearly stated? }\end{array}$ & $1,0.5,0$ \\
(Yes, nominally, No) & \\
(C4) Based on the findings, & $>80 \%=1,<20 \%=0$, \\
how valuable is the research? & in-between $=0.5$
\end{tabular}

\subsection{Overview of the Studies}

In [22], Yli-Huumo et al. reported an SLR study they conducted in 2015 externalizing the relevant research on blockchain technology from a broad perspective. In their findings, all their selected primary studies were published after the year 2012. Interestingly, blockchain had to wait three years after that date to reach the curiosity of the healthcare domain as we could not find any significant studies related to our research topic before 2016. However, since 2016, there has been an increasing number of publications. Figure 3 shows the publication year distribution of the selected primary papers.

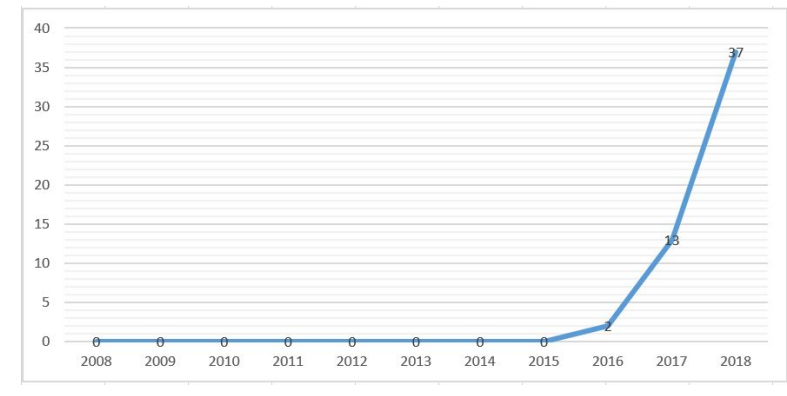

Fig. 3. Publication year of the selected primary papers.

Out of the 52 primary studies, 46 (88.4\%) were published in journals, 2 in conference proceedings (no more than $4 \%$ ), 3 in magazines $(5.7 \%)$ and one study was published in an Open Research publishing online platform. The studies' distribution was biased towards venues specialized in medical studies (23 papers, 44\%) in comparison to computer/software specialized venues (9 papers, $17.3 \%)$. The remaining 19 papers $(36.5 \%)$ were published in interdisciplinary venues relating the emerging information/software systems methods and applications to healthcare settings. One particular journal; namely "Journal of medical systems", hosted 12 (23\%) of the overall selected studies.

In reviewing the location of each author in each primary study, the geographical distribution of affiliations shows that blockchain technology has gathered research interest in the healthcare domain around the world, as 22 countries are represented. The most significant number of studies (23 papers, 44\%) were published with at least one affiliation from the United States. After this, the most common publication countries were China (10 papers, $19.23 \%$ ) and United Kingdom (5 papers, 9.6\%).

It is also interesting to observe how the research on blockchain is evolving from addressing healthcare generically towards more specialized aspects. Thirteen of the extracted studies discussed blockchain to address either a particular medical illness, a medical speciality or a medical procedure. Ten of these are published in 2018. We mapped these studies accordingly in Table 3:

TABLE 3

Mapping The Primary Studies to Medical Conditions / Specialties (RQ1.2)

\begin{tabular}{|l||c|}
\hline Paper & Medical Condition / Specialty / Procedure \\
\hline$[23],[24],[25]$ & Diabetes \\
\hline$[25]$ & Cardiac Failure \\
\hline$[26],[27]$ & Surgery \\
\hline$[28]$ & Radiology \\
\hline$[29]$ & Transfusion \\
\hline$[30]$ & Dermatology \\
\hline$[31]$ & Traditional Chinese Medicine \\
\hline$[32]$ & Essential Tremor (ET) - Neurology \\
\hline$[33]$ & Cancer \\
\hline$[34]$ & Pathology \\
\hline
\end{tabular}

When we conducted keywording at Step 5 of the selection process, we were able to identify five scenarios in which blockchain is being incorporated in the healthcare domain: 1) Sharing patient's healthcare records, 2) Medical Drug supply chain management, 3) Healthcare professionals' education and training, 4) Clinical research and experiments, and 5) Healthcare insurance claims processing. We decided to use these five scenarios as the first classification dimension to map the existing primary studies on blockchain in healthcare. The studies were mapped in Step 6 after each paper was entirely read. One study can be related to more than one scenario. We discuss these five scenarios further in Section 4. We also extracted a set of advantages (Section 5) and challenges (Section 6) in applying blockchain into each of these five scenarios and mapped the studies accordingly.

In addition, we identified that there were three non-mutual exclusive classes to which a primary study could be mapped to 1) providing literature discussion, 2) presenting an innovative blockchain-based implementation, and 3) providing validation of a blockchain-based application in practice. We used these three classes as a secondary classification dimension. While each of the 52 studies contributed to the literature discussions, only 33\% (17 studies) presented a blockchain-based implementation. We provide the list of these studies along with the blockchain frameworks used/proposed through the link: "https://bit.ly/2Kqx7vx". In 9 of the studies, the authors proposed their own blockchain framework, while in 5 of the studies the authors utilized Ethereum. None of these studies provided validation in real settings. A summarized map using these two dimensions is presented in Figure 4. For example, 13 studies which addressed the "medical supply chain" scenario provided a literature discussion on the topic, while 2 of these studies also provided a presentation of a system that was designed or implemented. As the figure also shows, none of the studies which discussed the "medical supply chain" scenario provided a validation of an implemented system in practice. The mapping details for the five scenarios are provided in Section 4. 


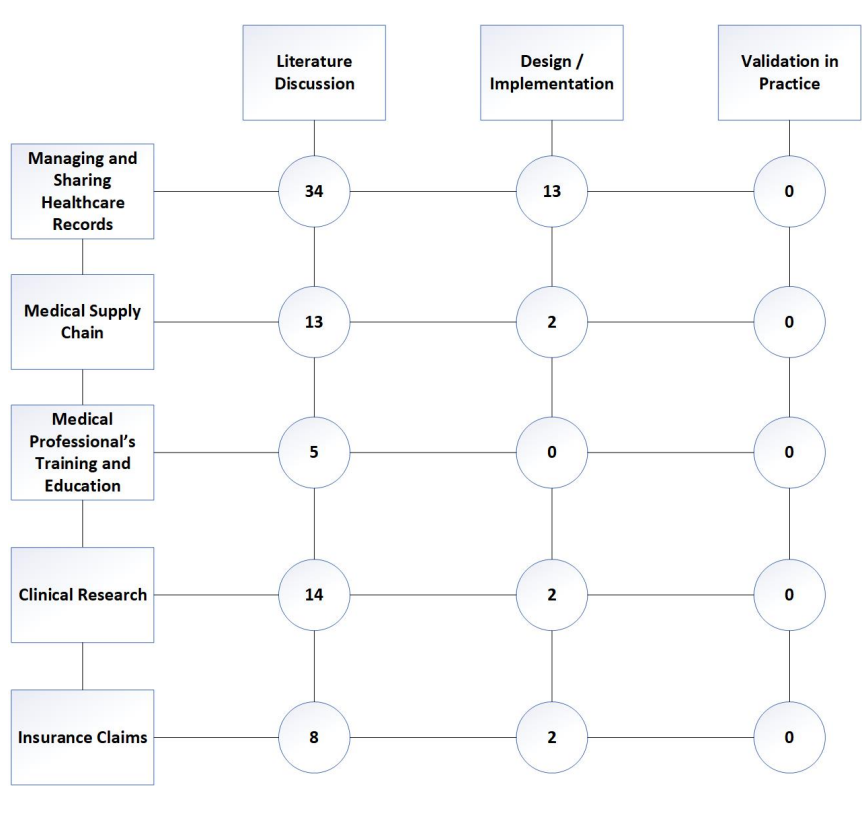

Fig. 4. Classification of the relevant papers.

\section{Adopted Scenarios of Blockchain HEALTHCARE (RQ1)}

After reading all the selected studies, we identified five primary scenarios on the potential usages of blockchain in healthcare, which we discuss in this section.

\subsection{Managing and Sharing Healthcare Records}

Numerous health systems globally are currently struggling to deliver core public health services - vaccinations, syndromic and disease surveillance, maternal and child health, and there is an urgent need to build system resiliency in anticipation to a rapidly growing number of significant stressors and threats to public health and social stability in the coming years. Healthcare data are the most valuable asset of any healthcare system's intelligence. Most of the time, these data are scattered across different systems and sharing them is influential for establishing effective and cohesive healthcare. For example, a patient could visit doctors in different hospitals for various symptoms; it would be beneficial for each doctor to see the patient's history. Under the current circumstance, a doctor could be rejected access to the data hosted by other establishment without an additional agreement for personal health information (PHI) sharing. Also, a centralized hosting location of data (e.g., cloud-based solution) can be a single point of a security attack [35]. Anecdotal evidence from recent years shows that healthcare data continued to be a lucrative target for data breaches [36], thus causing patients to be exposed to economic threats as well as possible social stigma and mental anguish [35].

Cross-institutional sharing of patient healthcare data is also complicated due to the demand for a high level of interoperability. As a consequence, data are not always accessible to the provider even though a patient could have granted access to that particular healthcare provider [23]. In an ideal world, patients should not only own but be able to control and share their data without compromising confidentiality. Polls show that about $80 \%$ of patients are willing to share their medical information [35], provided their confidentiality can be ensured.
With growing recognition of the distributed nature of health services and health records, attention has been increasingly focused on decentralized architectures and system interoperability. The most pressing need is for better communication, informationsharing, planning and coordination of service delivery between all members of distributed patient care teams. Blockchain, with its decentralization nature, can "liberate data from entrenched silos, empowering patients to securely own their data” [37].

Primary Studies: Incorporating blockchain to manage and share healthcare records was the most popular scenario among the extracted primary studies, with 34 studies contributing to the literature discussion, among which 13 presented an innovative application implementation in support of this scenario. Table 4 provides the mapping of these studies.

TABLE 4

Extracted Prime Studies on Blockchain for Managing and Sharing Health Records

\begin{tabular}{|c|c|c|}
\hline Paper & Provided literature discussion & Proposed application developed \\
\hline [38] & $\checkmark$ & \\
\hline [23] & $\checkmark$ & $\checkmark$ \\
\hline [39] & $\checkmark$ & \\
\hline [26] & $\checkmark$ & \\
\hline$[24]$ & $\checkmark$ & \\
\hline [40] & $\boldsymbol{V}$ & $\checkmark$ \\
\hline [41] & $\checkmark$ & $\checkmark$ \\
\hline [35] & $\checkmark$ & $\checkmark$ \\
\hline [28] & $\checkmark$ & $\checkmark$ \\
\hline [29] & $\checkmark$ & \\
\hline [42] & $\checkmark$ & \\
\hline [43] & $\checkmark$ & \\
\hline$[44]$ & $\checkmark$ & \\
\hline [45] & $\checkmark$ & $\boldsymbol{V}$ \\
\hline [46] & $\checkmark$ & \\
\hline [47] & $\checkmark$ & \\
\hline [48] & $\checkmark$ & $\boldsymbol{v}$ \\
\hline [49] & $\checkmark$ & \\
\hline [30] & $\checkmark$ & \\
\hline [50] & $\checkmark$ & $\boldsymbol{V}$ \\
\hline [51] & $\checkmark$ & $\checkmark$ \\
\hline [31] & $\checkmark$ & $\checkmark$ \\
\hline$[52]$ & $\checkmark$ & $\checkmark$ \\
\hline [25] & $\checkmark$ & \\
\hline [53] & $\checkmark$ & \\
\hline [33] & $\checkmark$ & $\checkmark$ \\
\hline [54] & $\checkmark$ & \\
\hline [34] & $\checkmark$ & \\
\hline [55] & $\checkmark$ & \\
\hline$[56]$ & $\checkmark$ & \\
\hline$[57]$ & $\checkmark$ & \\
\hline$[58]$ & $\boldsymbol{V}$ & \\
\hline [59] & $\checkmark$ & $\boldsymbol{V}$ \\
\hline [37] & $\boldsymbol{V}$ & \\
\hline
\end{tabular}

With the "decentralization", "provenance", "immutability" and "finality" blockchain's characteristics, most of the studies discuss the possibility of constructing a single shared ledger to store patients' medical data history for sharing or serving other purposes among stakeholders while mitigating the traditional threats to privacy due to the centralization facets of a traditional database 
or cloud environment.

The 13 developed platforms range from addressing generic health records (e.g., [40], [35], [48]) to more pragmatic ones targeting specific $\mathrm{p}$ opulation of $\mathrm{p}$ atients or $\mathrm{m}$ edical $\mathrm{s}$ pecialties. For example, in [23], Cichosz et al. presented a blockchain-based platform for sharing healthcare data of Diabetes patients among multiple entities. The platform employs NEM blockchain ${ }^{6}$ which supports multi-signatures enabling several entities administrative access and control of one data account. For example, the following steps occur when an entity (e.g., physician) prescribes a drug for a patient:

- The entity initiates the transaction.

- The patient accepts the transaction either manually or automatically (within certain rules).

- The transaction is encrypted and time-stamped.

- The encryption key is sent to the patient's multi-signature account (e.g., only entities with access will be able to read the prescription from the prescription ledger)

Finally, in order to change the multi-signature contract, a specified number of keys are required. The patient and a trusted party control the number of keys.

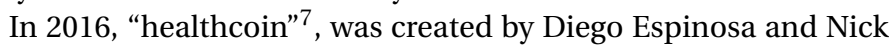
Gogerty. Healthcoin was the first platform based on blockchain to manage and reward Type-2 diabetes prevention. Users interact with the system by submitting their biomarkers into the blockchain. If the biomarker is an improvement, the system awards the patient with digital tokens: "healthcoins". Heathcoins can be applied towards government tax breaks and/or discounts on multiple fitness brands. Another emerging "healthcoins" are also in development (e.g., Universal Healthcoin $\mathrm{UHX}^{8}$ ).

Another instance of a specialized blockchain-based platform was presented in [28] describing a ledger that would enable patients free access to their medical image data in a secure manner without requiring a third-party administrator. While the actual radiological images are not stored inside the chain due to their large size; a block transaction links a public key to a uniform resource locator (URL) and establishes a source of medical imaging data. Using a unique identifier (UID), a transaction also establishes a source as the creator and a patient as the owner of a particular radiological study. In addition, a transaction enables the owner of that radiological study to authorize access to the particular radiological study to another party using the source endpoint URL. The system utilizes a "proof-of-stake" scheme to achieve consensus among the participating blockchain nodes.

Tung et al. presented a similar specialized blockchain-based system for dermatology in [30] to preserve dermatology-related images. Encrypted images can be stored via blockchain in that case though, with image ownership and locations encoded as transactions and in which patients can access and selectively share medical records using a private digital key. Tung et al. also pointed out the possibility of allowing machine learning algorithms on the top of the blockchain to access various images stored on the blockchain network to drive the optimization of computer-assisted analysis further.

In collaboration with Stony Brook University Hospital, Dubovitskaya et al. developed a framework on managing and sharing Electronic Medical Records (EMR) for cancer patient care [33].

\footnotetext{
6. https://nem.io/

7. https://healthcoin.com/

8. https://uhx.io/
}

Since blockchain eliminated the intermediate actors, the proposed framework aims to reduce cost, decrease the turnaround time for cancer patient EMR sharing, and improve medical care decision making.

Internet of Things (IoT)-based healthcare systems are also becoming widely popular to collect remote patient's data in various settings. For example, using analytics on aggregated data and then upon reporting this information to caregivers, an action is taken, such as shutting down a faulty medical device or changing drug dosage. While privacy is a significant concern when using such IoT systems [60], augmenting blockchain with sensors and IoTs technologies to support real-time patient monitoring has the potential to automatically notify, in a HIPAA compliant manner, any security vulnerabilities that are associated with remote patient monitoring. This application was discussed and presented through a system implementation based on private blockchain based on the Ethereum protocol in [41].

\subsection{Medical Supply Chain}

Although many of the the World Health Organization's (WHO) systems ${ }^{9}$ and a numerous number of global regulations (e.g., Drug Supply Chain Security Act (DSCSA) ${ }^{10}$ ) were designed to help on the protection of integrity of the medication supply chain by gathering data at each step of a medication's journey, the global trade in counterfeit medicines continues to be robust with remarkable risks to general public. Counterfeit drugs could contain inactive ingredients, incorrect dosage of active ingredients and/or potential contaminants causing possible adverse allergic reactions [61]. The use of poor quality antimicrobial medicines, for example, could cause significant treatment issues such as increased antibiotic resistance [62], which could result in higher mortality rates and the spread of highly resistant organisms. It is estimated that substandard or falsified antibiotics cause as many as 169,000 deaths of children worldwide from pneumonia [63].

Consequently, there is also a severe economic burden on society. It is estimated that this globalized pharmaceutical crime is now a multi-billion dollar industry threatening the lives of millions of citizens [64], [62]. It is speculated that in the United States, there is a relatively large unregulated 'grey market' of medicines populated by secondary wholesalers, traders, and re-sellers, where counterfeit drugs are sourced [65]. For example, the case of fake versions of the anticancer drug Avastin ${ }^{\circledR}$ in 2012 demonstrates when a falsified drug was possibly administered to thousands of US patients [66]. This type of pharmaceutical crime has more severely affected low and lower-middle-income countries. This situation is caused by the multiple parallel drug supply and delivery systems within a country creating a variation in quality and oversight [67]. It is estimated that 1 in 10 drugs in these countries are counterfeit [61]. For example, in 2003, the Philippines, $30 \%$ of inspected drugs were found with substandard or falsified drugs. Unfortunately, substandard/counterfeit versions of antimalarial treatments are not unusual (e.g. as high as $40-50 \%$ ) in many resource-poor regions, such as sub-Saharan Africa and Southeast Asia. Mitigating this crime could mean that hundreds of thousands of deaths and resistant strains could be prevented [68].

It is essential that drug Supply-chain visibility is carefully and consistently defined across industries. A multidisciplinary review of current and emerging digital solutions to combat the counterfeit

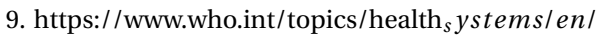

10. http://pdsaonline.org/dscsa-information 
drug incidences cited blockchain as one of five technologies with the potential to better establish drug supply chain root [69].

Primary Studies: We mapped 13 studies that provided literature on the potential usage of blockchain projects for the drugs supply chain (see table 5)

TABLE 5

Extracted Prime Studies on Blockchain for Drugs Supply Chain.

\begin{tabular}{|l||c|c|}
\hline Paper & Provided literature discussion & Proposed application developed \\
\hline$[70]$ & $\boldsymbol{V}$ & $\boldsymbol{V}$ \\
\hline$[69]$ & $\boldsymbol{V}$ & \\
\hline$[38]$ & $\boldsymbol{V}$ & \\
\hline$[71]$ & $\boldsymbol{V}$ & \\
\hline$[72]$ & $\boldsymbol{V}$ & \\
\hline$[73]$ & $\boldsymbol{V}$ & \\
\hline$[74]$ & $\boldsymbol{V}$ & \\
\hline$[6]$ & $\boldsymbol{V}$ & \\
\hline$[75]$ & $\boldsymbol{V}$ & \\
\hline$[46]$ & $\boldsymbol{V}$ & \\
\hline$[32]$ & & \\
\hline$[58]$ & & \\
\hline$[76]$ & & \\
\hline
\end{tabular}

Only two papers [70], [32] presented an actual application based on blockchain technology that intended to trace the drug pathway starting from the manufacturer to the consumer. In [70], for example, the application is being developed as a Distributed Application (DApp) that will run on smart contracts, and employing Swarm $^{11}$ as the Distributed File System (DFS). Once tested, the application is intended to be deployed in the Philippines with five starting nodes for the prototype (e.g. one for each participant in the traditional drug distribution model): manufacturer, wholesaler, retailer, Food and Drug Administration (FDA), and a consumer portal website. With such an application, a consumer will receive a code (to be scanned with a mobile phone camera) along with drug product purchased. After scanning the code, the consumer is directed then to the portal, which displays the distribution history of the drugs. The system is capable of detecting six anomalies along the chain: 1) Missing nodes, 2) Incomplete distribution chain, 3) Invalid node certificates, 4) Unregistered products, 5) Discrepancies in Primary data point (e.g., drug-related date) and 6) Timestamp anomalies.

Augmenting blockchain with Internet of Things Application (IoTA) to track medical drugs and patients' data is presented through a solution in [32]. With the help IoTA, cost-free transactions can be added to the network with easier trust schema. In the proposed solution, every participant node in the network, which is making a transaction, also actively participates in the consensus. The solution utilized a newly designed trinary hash function called Curl, which is quantum immune.

\subsection{Medical Professionals' Training and Education}

The sphere of the healthcare education is rapidly evolving as it needs to accommodate advances in biomedical sciences, new regulatory policy, technological advancements and improvements in learning theory. Hence, health profession educators face the challenge to adapt educational content to include the state of the art advancements in the field [77]. Recently, digital platforms

11. https://www.swarm.space/ (e.g., massive open online courses (MOOCs), social media-based education) have become effective forces to shape higher education in general, including the medical curricula [77]. On the other hand, the structure of the world wide web, where most of these digital platforms are being hosted, does not intrinsically allow for traceability and verification to the source's identity of the medical content that is being shared. In essence, the accuracy of the content found on the internet is questionable. This lack of judgment cannot be tolerated in a highly regulated discipline such as medicine. Healthcare education platform based on a blockchain has the potential to provide improved tracking of healthcare educational content and the creators.

With the wide range of sources to acquire education, employers across many disciplines are not just focused on degrees earned to demonstrate one's abilities; consequently, digital credentials are also a factor in assessing someone's ability [78]. While, this is true in some domains such as engineering; it may not be accepted yet as readily in healthcare, but as discussed in [42], maintaining digital credentials within a permissioned blockchain network can open the appetite to start considering them in healthcare.

Primary Studies: We mapped five studies to the education/training scenario (See table 6). One particular study described the scope of using blockchain for medical professionals' education in Russia [6], while the remaining four studies were not restricted in their discussion to a particular location.

Although none of the studies provided an actual implementation or validation in practice, the potential usage of blockchain was pointed out to address the traceability of educational content and digital credentials. As described in [77] on tracing contents to the sources: "a medical school's blockchain-based curriculum could link blocks of curricula to the faculty responsible for their course or block. Curriculum evaluation would then take place in the blockchain system, where the highest utilized, most validated, and better-evaluated deliverables could be easily identified, and the authors rewarded accordingly". As learners' transactions will remain connected in a chain, it is also feasible to quantify the educational impact an educator has on multiple generations of learners.

Another unique opportunity is using blockchain to manage and share a physician's credentials. As described in [39]: a physician can submit copies of their degrees, residency completion, online certificates, among other documents once to the administrative authority on the blockchain. Each credentialing committee would have a permissioned access to this digital ledger to submit and review records that have been collected in digital format.

TABLE 6

Extracted Prime Studies on Blockchain for Healthcare Profession's Education.

\begin{tabular}{|l||c|l|}
\hline Paper & Provided literature discussion & Proposed application developed \\
\hline$[77]$ & $\boldsymbol{V}$ & \\
\hline$[6]$ & $\boldsymbol{V}$ & \\
\hline$[42]$ & $\boldsymbol{V}$ & \\
\hline$[75]$ & $\boldsymbol{V}$ & \\
\hline$[39]$ & $\boldsymbol{V}$ & \\
\hline
\end{tabular}

\subsection{Clinical Research}

The credibility of scientific results from clinical trials may be eroded by a range of concerns including incomplete data, endpoint 
switching and publication bias [79]. During clinical trials, it is estimated that $85 \%$ of research resources are wasted due to findings being exaggerated or even entirely false. While these concerns have contributed to poor perceptions regarding the advantages and risks of treatments, the legislative intervention has seen limited success as well in improving the situation. For example, while U.S. FDA regulations require that the data of all clinical trials be made available, a recent study suggests that fewer than half of trials comply [80]. A blockchain-based solution for trials record management may, therefore, provide an alternative with which to address these concerns. The "provenance" feature of blockchain can save the cost of the "Source data verification" which is currently estimated at $20 \%$ to $30 \%$ of the overall clinical trial budget [43]. Blockchain's ability to store transactions anonymously while still enabling participants to authenticate themselves when required is also a critical feature that will benefit the clinical research. Entities can use unique key pairs to manage each study, while the private keys are kept secure. Consequently, patients will not be identified by analyzing a blockchain recorded transaction. However, still, when necessary, any entity can prove ownership of a public key by merely signing a message with the corresponding private key [28].

Primary Studies: We mapped 14 studies that provided literature on the potential usage of blockchain projects to support clinical research (see table 7). For example, in [73] the authors described how blockchain could "increase transparency and crossinstitutional visibility of the process of unfinished trials because it will be much easier to share information that is not confidential (for example, the overall number of participants), especially with smart contracts". In [72], a discussion on the positive impact of blockchain on the clinical drug supply chain was provided. This impact is linked to the traceability improvement of medications from active pharmaceutical ingredient to patient while facilitating the gathering of patient-level data in a HIPAA-compliant manner.

The authors of [43] provided a scenario discussion on how blockchain can increase the credibility of the clinical trials data since the technology can be used as proof that the trial's data existed at a particular stage and was not forged.

TABLE 7

Extracted Prime Studies on Blockchain for Clinical Research.

\begin{tabular}{|l||c|c|}
\hline Paper & Provided literature discussion & Proposed application developed \\
\hline$[26]$ & $\boldsymbol{V}$ & \\
\hline$[38]$ & $\boldsymbol{V}$ & $\boldsymbol{V}$ \\
\hline$[28]$ & $\boldsymbol{V}$ & \\
\hline$[72]$ & $\boldsymbol{V}$ & \\
\hline$[81]$ & $\boldsymbol{V}$ & \\
\hline$[82]$ & $\boldsymbol{V}$ & \\
\hline$[73]$ & $\boldsymbol{V}$ & \\
\hline$[46]$ & $\boldsymbol{V}$ & \\
\hline$[79]$ & $\boldsymbol{V}$ & \\
\hline$[83]$ & $\boldsymbol{V}$ & \\
\hline$[27]$ & $\boldsymbol{V}$ & \\
\hline$[55]$ & $\boldsymbol{V}$ & \\
\hline$[58]$ & & \\
\hline$[37]$ & & \\
\hline
\end{tabular}

\subsection{Insurance Claims}

An unfortunate reality is that some of the health insurance claims are fraudulent and very costly to the healthcare industry. It is estimated that insurance fraud in the healthcare industry costs tens of billions of dollars annually. Since 2008, fraud and errors in the healthcare industry have risen 25 per cent to 6.9 per cent total. [84]. The immutable audit trail that blockchain provides is a key feature that can benefit the healthcare insurance industry. Traditional database systems used in healthcare support create, read, update, and delete functions, blockchain with the "immutability" feature only supports create and read functions. Thus, blockchain's unchangeable ledger is more appropriate to record critical information, such as insurance claim records. Besides, the "provenance" feature implies that the origins of the assets are traceable and can be verified which will increase the reusability of the insurance transaction data. There is so much support that the blockchain technology is much more effective to manage critical digital assets (e.g., insurance claims, patient records, etc.).

Primary Studies: We mapped eight studies that provided literature on the potential usage of blockchain projects to support the health insurance industry (see Table 8).

TABLE 8

Extracted Prime Studies on Blockchain for Healthcare Insurance.

\begin{tabular}{|l||c|c|}
\hline Paper & Provided literature discussion & Proposed application developed \\
\hline$[42]$ & $\boldsymbol{V}$ & \\
\hline$[85]$ & $\boldsymbol{V}$ & $\boldsymbol{V}$ \\
\hline$[51]$ & $\boldsymbol{V}$ & \\
\hline$[86]$ & $\boldsymbol{V}$ & \\
\hline$[38]$ & $\boldsymbol{V}$ & \\
\hline$[55]$ & $\boldsymbol{V}$ & \\
\hline$[58]$ & $\boldsymbol{V}$ & \\
\hline$[37]$ & $\boldsymbol{V}$ & \\
\hline
\end{tabular}

Only [85] and [51] provided actual blockchain-based application to support insurance claim storage. In [85], the authors proposed MIStore. MIStore adopts the Practical Byzantine Fault-tolerance (PBFT) algorithm to guarantee consensus of the blockchain. With such an application, a hospital may directly store confidential insurance data in the blockchain, while an insurance company may send a query directly to the chain. Because of combining the needs of the insurance company with the hospitable with blockchain, the system becomes more efficient, e.g., decentralization (removing the middleman), tamper-resistance and record-nodes to enable user verification. Additionally, when the insurance company performs a query, the specified servers can perform homomorphic encryption computations on the data before a returned response. If the insurance company can collect a predetermined threshold $(t)$ number of correct responses from the server, only then will the insurance company be able to recover the correct patient's spending data history. The other blockchain-based insurance application [51] is a secure Cloud-Based EHR System which uses Attribute-Based Cryptosystem and Blockchain technology. They provided a brief demonstration of how the system may provide transparency and better service of the medical insurance claims.

\section{Key Benefits of InCORPorating BlockChain in HealthCARE (RQ2)}

Currently, medical data are not immune to concerns about misuse or inefficient use of. With the key features of blockchain in com- 
parison to traditional distributed databases, we identified the four key benefits $t$ hat $t$ hese $b$ lockchain $f$ eatures a dd $t$ o $t$ he extracted scenarios: 1) improved availability; 2) improved transparency; 3) improved security; and 4) improved performance. We provide a mapping of the extracted primary studies to the four critical advantages in Figure 5 and a comprehensive discussion herein.

Availability: Data generated from different sources, including wearable devices, can be directly stored into a medical chain which can be used then by patients to easily review their medical record history even if they are housed in distributed storage locations. "Because data is stored on a "decentralized" network, there is no single point of attack" [87], which reduces the risk of unavailability to patient record keeping. The patients can then, selectively and securely, share access of their own with any trusted third party they wish. "[Provider, health-related services and medical goods have the benefits of] p atient d ata a ccessible from multiple silos [based on Blockchain virtual ledger]." [86], which provides enhanced accessibility. With blockchain, healthcare providers also have access to near real-time data: "Blockchain would ensure continuous availability and access to real-time data. Real-time access to data would improve clinical care coordination and improve clinical care in emergency medical situations. Real-time data would also allow researchers and public health resources to rapidly detect, isolate and drive change for environmental conditions that impact public health. For example, epidemics could be detected earlier and contained." [88]. The immutability feature also implies the availability of the entire history of health records.

Improved Transparency: The provenance feature of the blockchain implies that any additions to the blockchain are visible to all of the patient's network members. Given that data are immutable, any unauthorized modifications $\mathrm{c}$ an b e trivially diagnosed. In the context of counterfeit medications, for example, a blockchain application will be able to: (1) track pharmaceutical raw materials (e.g. manufacturer) to finished p roduct (e.g. enduser) in an immutable and shared distributed ledger; (2) provide detection of counterfeit drugs in the drug supply chain given that blockchain participants verify the data in the chain, possibly with the integration of IoT anti-counterfeit devices; and (3) serve as an open standards technology that will ultimately further improve the quality of information sharing across unrelated databases with multiple participants in the drug supply chain. This type of blockchain application has the potential to transform the drug supply chain into a shared, transparent, and trustworthy open data architecture that could include multiple participants and jurisdictions. "In the existing solutions, there is still a central authority that can be compromised and documents that can be faked...If [the current solutions] can be modified with b lockchain enabled anti-tampering capabilities during manufacturing, the supply and dispensation system could make drug counterfeiting a non-issue." [87].

Medical worker training is ongoing, thus blockchain has the potential to be used used to oversee by recording and verifying of the skill set training and education of medical personnel. The provenance feature allows tracing educational content to its sources. Eventually, this can evolve into a global shared repository of credentials of healthcare professionals where the digital identity of a physician along with his/her credentials, can be shared and traced.

Another critical benefit of $\mathrm{b}$ lockchain is the s torage of health claims as it comprises sensitive data that needs protection and verification. $\mathrm{B}$ lockchain $\mathrm{c}$ an a lso i mprove $\mathrm{t}$ he e fficiency of the insurance claim process as it can provide near real-time claim processing, verification of benefit eligibility and provide preauthorization for providers. In other words, payers and providers can share encrypted patient identifiers, claims and other health plan information within a blockchain.

Doctors are facing problems with the existing large file-sharing options [89]. File sharing improves patient care, as important information is known and coordinated. For example, the New York Times (2014) explains the difficulty medics face in an emergency when trying to send digital records containing patient information. Blockchain technology can also be beneficial in sharing large medical files.

Improved Security: Medical information is sensitive data that needs to be kept private and secure. With data breaches on the rise, it is imperative to ensure the security and privacy of all medical files including but not limited to test imaging and insurance data. In 2015, the Health and Human Services (HHS) office reported that over 113 million individuals were affected by protected health information breaches. Most (99\%) were hacking victims, while only $1 \%$ suffered from other forms of a data breach (e.g. improper disposal or unauthorized access/disclosure). HHS also reported that network servers (107 million) and EHRs (3 million) are the leading sources of information breaches.

Because of the "decentralization" feature in blockchain, no single data point can be hacked to steal patient records. Decentralization of the data ledgers provides an immutable history of events. Depending on if the data is private or public, the history can be made transparent. In the case of private data (medical records) data can be obfuscated by calculating and storing the hash number. Blockchain data is encrypted and requires the patient's private key to be decrypted. A hacker will not be able to read the patient data even upon access. A fortunate side effect of the higher security is a higher patient confidence in consent recording systems: "Patients are able to add consent statements at any point in their care journey - confident that the blockchain will hold them securely." [90]. This situation can eventually lead to an increased level of data sharing which is essential for a more efficient healthcare system: "Utilization of the...health blockchain...has the potential to engage millions of individuals, healthcare providers, healthcare entities and medical researchers to share vast amounts of genetic, diet, lifestyle, environmental and health data with guaranteed security and privacy protection." [88]. In the context of claim processing, "[Member/patient have the benefits of] less likelihood of hacking of...financial information [based on Blockchain mechanism]" [88], which increases the security of patient medical insurance information.

Improved Performance: The "decentralization" of a blockchain improves performance bottlenecks that result from frequent network communication. Integrating IoT with blockchain as proposed in [41] can also reduce the need for doctors appointments. Blockchain technology would provide an active feedback loop in such a scenario to provide real-time remote patient monitoring and medical interventions that would allow delivery of notifications to all involved parties automatically.

\section{Key Challenges OF Incorporating BLockchain IN HealthCARE (RQ3)}

Despite the above advantages, in its current state, blockchain will not offer the complete answer for all the challenges of the discussed scenarios. More specifically, we extracted the following challenges: 


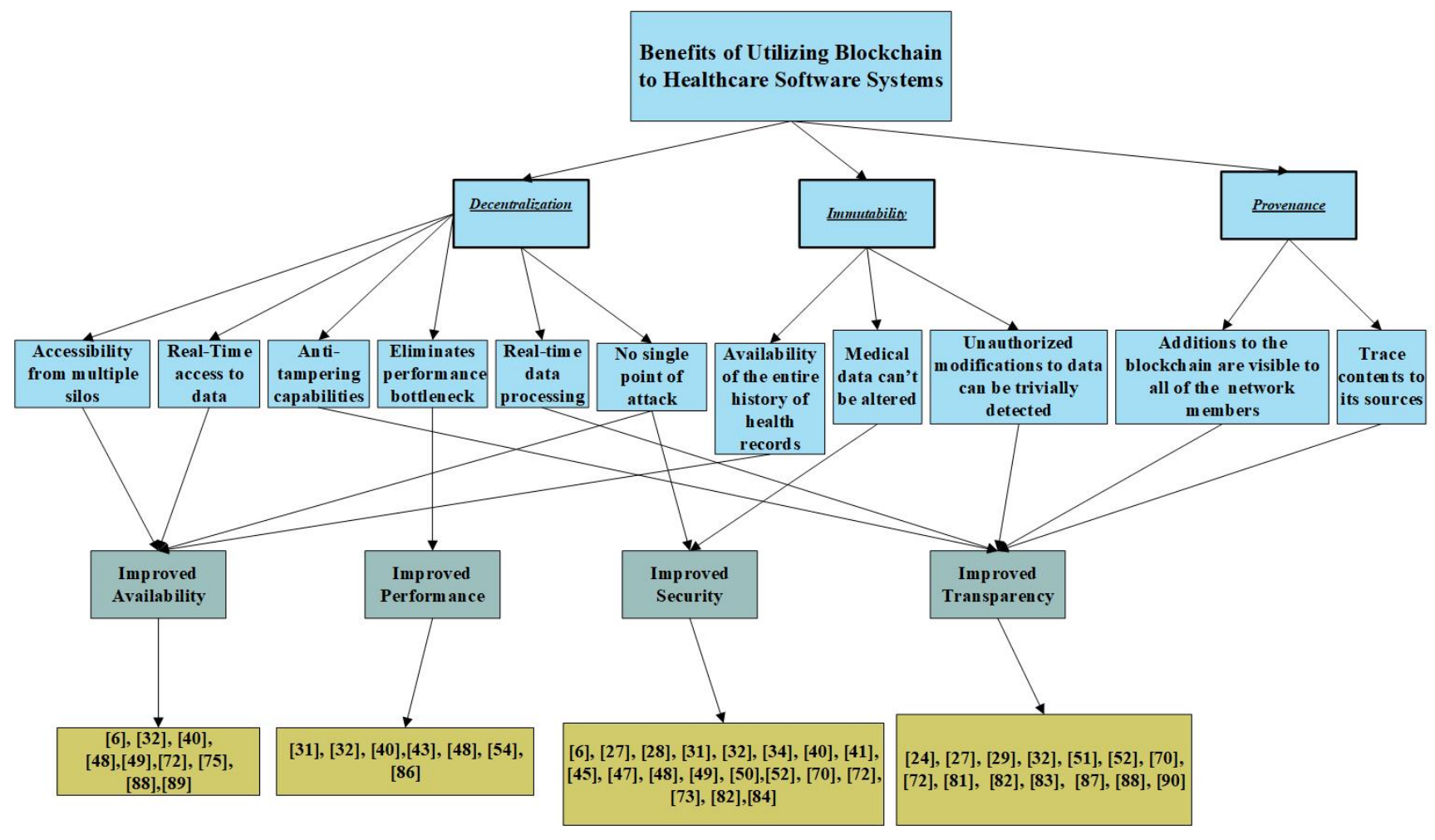

Fig. 5. Mapping of the Key Benefits of Incorporating Blockchain in Healthcare Software Systems

1) Scalability and performance, 2) Usability, 3) Secure identification and 4) Lack of Incentives and willingness to adopt. We provide a mapping of the extracted primary studies to the four critical challenges in Figure 6 and a comprehensive discussion herein.

Scalability and Performance: While we could envision the use case of storing the EHRs within a blockchain, storing large medical files (e.g. X-ray and ECG), are too large for direct storage. This challenge was discussed in [38], [23], [43] and [39].

In addition, within a blockchain deployment, the "decentralization", "consensus" and "provenance" features imply that all blocks should be stored on every participating client node within a system. As the size of data will be on constant increase, a demand on every participating node will also increase in order to provide the necessary scalability. To illustrate this scalability issue, a miners full participation in the Bitcoin network requires the miner to download the entire Bitcoin ledger, which totalled over 184 gigabytes at the end of Q3 2018. Also, the maximum transaction validation within the Bitcoin network is at seven transactions/second, which increases the possibility of a performance bottleneck. The blockchain-based platform that maintains a significantly larger volume of data has yet to be proven in production settings as of yet [38]. In [23], a possible solution to this challenge is to store an extensive collection of medical data off the chain in a data repository called a data lake. This would still be secure as the blockchain layer would enforce the access control policy. In this framework, "the patient would still have control of who has access to the personal data in the data lake because the data would not be readable without the decryption key, which is stored on the patient's blockchain account" [23].

Usability: The cryptographic concepts of Blockchain transactions will be unfamiliar to most people. In the context of medical records sharing, the proposed schemes from the extracted studies require patients to manage their key pairs (public/private) in order to provide cryptographic signatures, and authorize access to their medical data, as we discussed. That said, the structural complexity of managing the keys should be concealed behind web and/or mobile application with a user-friendly interface [23]. However, this also opens the door to a potential security threat that we will discuss next. Self-governance poses another challenge if the patient is unable to approve the necessary access permits. This may occur from merely the loss of personal keys to an acute critical illness such as Alzheimer's disease. Also, in case of an emergency, the medical data should be accessed to medical staff by invoking a procedure using a trusted party (e.g., governmental organization, close relative, etc.).

The MIT Media Lab examined digital certificate implemented with the blockchain technology. Some lessons learned in its first experiments include: "it is much more difficult to manage public/private keys to authenticate both issuer and recipient, hence establishing a wallet that maintains certificates; as Bitcoin holds money, may be an alternative." [38].

Secure Identification: In healthcare, the need to match patients to their care records across different healthcare provider backends (hospital EHRs, HIEs, labs, etc.) is critical and non-trivial. In the US, the Centers for Medicare \& Medicaid Services (CMS) has placed much greater emphasis on healthcare interoperability with its "Promoting Interoperability Program", intended to make patient records access to/from stakeholders easier. Startups, e.g. digitalhealthcare.io ${ }^{12}$, is spending resources trying to help resolve some of these very same interoperability issues. These innovations and policy changes, while positive, do not reach far enough upstream to resolve the question: how do we know who is accessing these patient records in the first place? Who is the real endpoint? It is

12. https://digitalhealthcare.io/ 


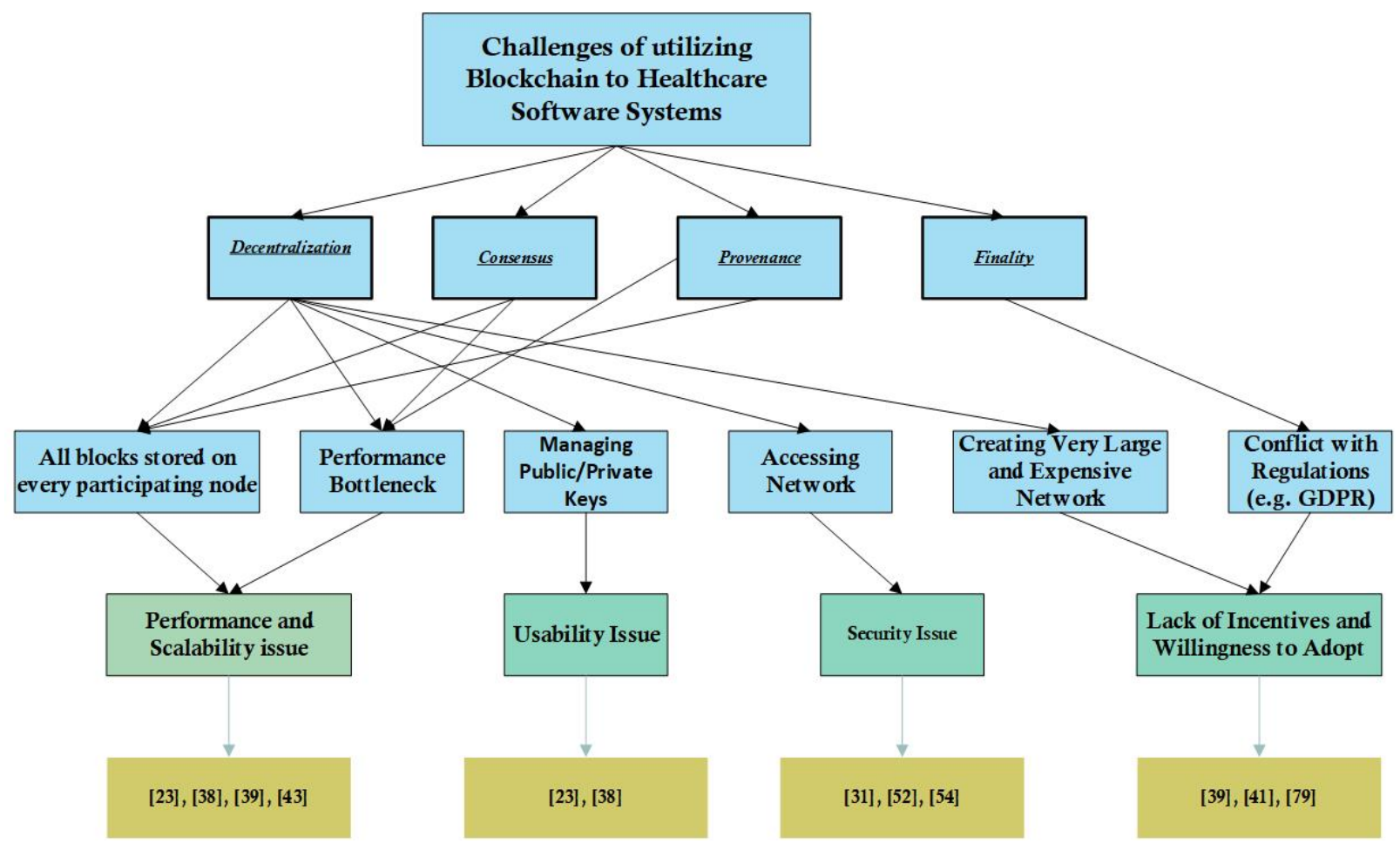

Fig. 6. Mapping of the Key Challenges of Incorporating Blockchain in Healthcare Software Systems

all about identity, and, within the domain of blockchain technologies, identity management is an essential component. Through a variety of related technologies, we can associate a user's device (e.g., smartphone) to the uniquely-signed and crypto-secure digital wallet as discussed above. So complete is this technology that we can ascertain that it is John Doe's smartphone that is just extracted tokens from Jane Doe's digital wallet. However, smartphones and digital wallets are not people. They are proxies at best and are prone to failure, get stolen, and sometimes just plain get lost. The integration of unobtrusive biometrics that does not infringe on privacy regulations on the top of a blockchain could be a start to better defining the effect of the unidentified, uninsured patient on overall healthcare expenditures. The most widely accessible biometrics already in circulation include fingerprints, facial recognition, retinal scanners, and heart-based readings (crude EKG, heart rate monitors). Some have alluded to a combo for a valid ID. There remain significant hurdles to overcome between establishing privacy regulation on the use of the biometric: defining related policies on reimbursement, and the preceding research that would need to take place regarding the effect of the uninsured on hospital closures - before any project could get underway in practice.

Lack of Incentives and Willingness to Adopt: Creating an extensive network of connected nodes is creating a major monetarily driven challenge. For example, very recently EHR systems were built and cost tens of billions of dollars, and very recently, many large health systems, incentivized by the governments worldwide, there have been millions of dollars invested building commercial EHR systems [41]. To request for an immediate replacement of the current record system with a digital ledger is seems to be irresponsible spending on behalf of taxpayers and will be a disservice to the medical field.

Conversely, for success at maintaining the integrity of the consensus algorithm and providing the minimum number of validations signatures, it is crucial to have a sufficient number of nodes online at any time. Instead, to improve this situation, blockchain would play a more supplemental role and not completely replace the current system. For example, each node could display a minimal amount of description data about a specific patient and procedure performed while keeping test results off of the blockchain. The link embedded in a block would act as a pointer to an off the blockchain API that would allow access to the test results.

The "finality" characteristic of the blockchain can also conflict with existing legislation such as the new European GDPR ${ }^{13}$ which aims to give all citizens the ability to govern their over their data including the right for every citizen to request an institution to delete his/her personal data.

In the context of the healthcare professionals' education, one additional challenge for the use of blockchain in academic institutions will be registrars that will fear losing money and control of the process [39]. Schaffhauser compares this to a registrar at Carnegie Mellon University [78], who "likens the future acceptance of blockchain to the previous adoption of the National Student Clearinghouse". while The clearinghouse was not instantly adopted, it is currently being used by approximately 4000 universities.

\section{Discussions and Roadmap for Future Re- SEARCH}

Blockchain comprises a cutting-edge technology that delivers a high precision of traceability for the artefacts in which it is applied and domains that are accordingly supported by it. Particularly considering healthcare domain, blockchain has the potential to support a holistic view and awareness of the entire lifecycle of medicines, medical equipment, health supplies, besides reinforcing

\footnotetext{
13. https://eugdpr.org/
} 
the value of health records and potentializing the ownership of the medical history to the patients through unified registers. This can avoid replication of clinical trials, reducing corruption by tracking the entire supply chain, and favouring economy by providing realtime transparent processing for the medical data. A large-scale registry of the entire health system of a country could facilitate accountability and diagnosis of critical points of improvement for the supply chain, besides maybe enabling a fast and precise identification of $\mathrm{n}$ ovel $\mathrm{h}$ uman $\mathrm{t}$ hreats, s uch a s $\mathrm{n}$ ew $\mathrm{v}$ iruses and infections, such as H1N1 that was detected a decade ago and demanded a rapid action from public authorities to reduce human losses.

This literature review suggests that we are still at the beginning of the road toward commercialization of blockchain technology and its application in the healthcare field. T hirty-three p er cent of the overall primary studies provided an actual implementation for blockchain systems in the sphere of healthcare. Twenty-five per cent of the studies provided an implementation for healthcare records management, making it the most popular scenario. While none of the extracted studies provided an actual validation on a large-scale in practice, our review of the grey literature also turned up several additional examples of prototypes, presentations, use cases, and initiatives that are currently under development/validation phase worth mentioning. One implementation example is Guardtime ${ }^{14}$, a blockchain-based framework to validate patient identities ${ }^{15}$. Guardtime was created by a Netherlands based data security firm in p artnership with the government of Estonia. A smartcard that links electronic health records (EHR) data to an individuals blockchain-based identity was issued to all citizens. A second EHR-related implementation is MedRec ${ }^{16}$ [91]. MedRec is a project that was initiated between MIT Media Lab and Beth Israel Deaconess Medical Center. This platform provides a decentralized approach in which the permissions, data storage location, and audit logs are maintained in the blockchain, while all healthcare information remains in the already existing EHR systems. This implementation will require additional software components to enable true software interoperability. The system was tested as a proof of concept. A third EHR project is UK's first trial of blockchain in healthcare to create and support EHRs which commenced on July 2018 as a prototype at a southwest London general practice group [44] allowing Medical-chain to gather feedback from doctors and patients so that they will use to refine the s ystem before its global launch. Well-known companies, such as Deloitte and Accenture, have been involved in designing blockchain enabled technology for healthcare data and medical record management.

For pharmaceutical supply chain-related blockchain activities, one project by iSolve, LLC $^{17}$ simulates a theoretical supply chain using blockchain to track medication. Chronicled, Inc. ${ }^{18}$ built another prototype combining NFC embedded adhesive seals that are registered and verified on a blockchain. A multistakeholder BlockRx project $^{19}$ is also being developed to pilot blockchain technology in the pharmaceutical sector.

This literature review also demonstrated that there are few significant research gaps still exist in utilizing blockchain systems in

\footnotetext{
14. https://guardtime.com/

15. https://cointelegraph.com/news/estonian-government-adoptsblockchain-to-secure-1-mln-health-records

16. https://medrec.media.mit.edu/

17. https://isolve.io/

18. https://www.chronicled.com/

19. http://www.blockrx.com/
}

healthcare. Swan [15] presents seven technical challenges and limitations for the adaptation of Blockchain technology in the future: 1) Throughput, 2) Latency, 3) Size and Bandwidth, 4) Security, 5) Usability, 6) Wasted Resources and 7) Versioning, hard forks, multiple chains. We could find that the extracted studies in this review established an early discussion on the first five challenges, but we could not identify any discussion regarding wasted resources and versioning, hard forks, multiple chains in the current literature. This is a significant research gap, which requires more research in the future. While the sizes of the current blockchain applications are relatively small, in the future, if blockchain has to handle billions of transactions, then more research needs to be conducted to ensure horizontal scalability.

The second research gap is that the majority of current research (33\% of the studies) is discussed concerning the Ethereum environment, rather than any other Blockchain environments. There are currently over a hundred of existing blockchain technologies ${ }^{20}$ and it is necessary to research the possibilities of using other blockchain projects because it can reveal and produce better alternative models and scenarios for doing different sort of healthcare transactions.

\section{CONCLUSION AND STUDY LIMITATION}

To obtain a full understanding of the state of the art research on blockchain technology and how blockchain is being utilized in the sphere of healthcare, we mapped all of the relevant research by following the SLR process [8]. The ultimate goal of the systematic mapping study presented in this paper was to examine the current status and research topics of blockchain technology in healthcare, along with the key benefits and challenges this technology will bring to the field. We extracted and assessed 52 primary studies. Our findings from the extracted scenarios in this review show that blockchain has generated a lot of interest and attention to be implemented as a platform to improve the authenticity and transparency of healthcare data through the five extracted scenarios despite the need to be moderated with attention to the essential challenges uncovered in this review. Given these results, we can provide recommendations on future research directions of blockchain technology based on the status of the state of the art research.

The primary limitations of any SLR are related to publication and selection bias, possible inaccuracy in the data extraction process and data misclassification [92].

Publication bias refers to researchers focusing and publishing only the positive results of their research and are less likely to publish the negative results given that negative results are more challenging to be published in peer-reviewed publications [92]. To address this issue, we utilized the PennState LionSearch tool to look for manuscripts. This integrated search engine provides results integrated from over 950 database/search engines, including over 80 databases/search engines for healthcare/medicine discipline and over 15 for computer/software/ information science and engineering. This search resulted in an increased number of papers to review, which implies there was an increase in finding papers with published negative results.

Selection bias refers to the "distortion of statistical analysis owing to the criteria used to select the publications" [92]. Our review encompassed only articles that primarily focused on blockchain addressing the healthcare domain. We carefully followed our research questions to define keywords for the search in the databases.

20. https://en.bitcoinwiki.org/wiki/Blockchain roject $_{L}$ ist 
In addition, we utilized a multistage process where each stage of the study was assessed by at least two researchers using the established inclusion/exclusion criteria.

Inaccuracy in data extraction and misclassification refer to the possibility that information is extracted and interpreted differently by different reviewers [92]. To address this, the full text of each extracted study was reviewed thoroughly by at least two authors. To review the agreements and disagreements raised in the assessments, we conducted several face-to-face consensus meetings along the process. After reading the research, we also updated the categories as well as created new categories to match our unanticipated findings. The data extracted from the relevant papers resulted in a map created systematically of the clustered categories formed.

\section{REFERENCES}

[1] H. K. Patil and R. Seshadri, "Big data security and privacy issues in healthcare," in 2014 IEEE International Congress on Big Data. IEEE, 2014, pp. $762-765$.

[2] H. Mack. (2017) Remote patient monitoring market grew by 44 percent in 2016, report says. https://www.mobihealthnews.com/content/remotepatient-monitoring-market-grew-44-percent-2016-report-says.

[3] J. G. Daniel and M. Uppaluru. (2017) New reimbursement for remote patient monitoring and telemedicine. https://www.cmhealthlaw.com/2017/11/new-reimbursement-for-remotepatient-monitoring-and-telemedicine/.

[4] The Office of the National Coordinator for Health Information Technology (ONC), U.S.A., "The value of consumer access use of online health records," 2015.

[5] V. Patel, W. Barker, and E. Siminerio, "Trends in consumer access and use of electronic health information," ONC Data Brief, vol. 30, 2015.

[6] K. Koshechkin, G. Klimenko, I. Ryabkov, and P. Kozhin, "Scope for the application of blockchain in the public healthcare of the russian federation," Procedia Computer Science, vol. 126, pp. 1323-1328, 2018.

[7] M. Walport, "Distributed ledger technology: beyond block chain. UK government office for science, london," UK, Technical report, Tech. Rep., 2016.

[8] B. Kitchenham and S. Charters. (2007) Guidelines for performing systematic literature reviews in software engineering. https://goo.gl/9aXfJ7.

[9] M. Kassab, J. DeFranco, T. Malas, V. V. Graciano Neto, and G. Destefanis, "Blockchain: A panacea for electronic health records?" in International Workshop on Software Engineering for Healthcare in conjunction with ACM/IEEE International Conference on Software Engineering, 2019.

[10] M. Kassab, J. DeFranco, T. Malas, G. Destefanis, and V. V. G. Neto, "Investigating quality requirements for blockchain-based healthcare systems," in Proceedings of the 2nd International Workshop on Emerging Trends in Software Engineering for Blockchain. IEEE Press, 2019, pp. 52-55.

[11] S. Nakamoto, "Bitcoin: A peer-to-peer electronic cash system," 2008.

[12] A. M. Antonopoulos, Mastering Bitcoin: unlocking digital cryptocurrencies. " O’Reilly Media, Inc.", 2014.

[13] Z. Zheng, S. Xie, H. Dai, X. Chen, and H. Wang, "An overview of blockchain technology: Architecture, consensus, and future trends," in 2017 IEEE International Congress on Big Data (BigData Congress).

[14] M. Gupta, "Blockchain for dummies," IBM Limited Edition, US, 2017.

[15] M. Swan, Blockchain: Blueprint for a new economy. O'Reilly Media, Inc., 2015.

[16] M. Belinky, E. Rennick, and A. Veitch, "The fintech 2.0 paper: Rebooting financial services, in http://www. oliverwyman. com/content: dam/oliverwyman/global/en/2015/jun," The_Fintech_2_0_Paper_ Final_PV.pdf, 2015.

[17] E. Hernandes, A. Zamboni, S. Fabbri, and A. D. Thommazo, "Using GQM and TAM to evaluate StArt-a tool that supports systematic review," CLEI Electronic Journal, vol. 15, no. 1, 2012.

[18] T. Dybå and T. Dingsøyr, "Empirical studies of agile software development: A systematic review," Information and software technology, vol. 50, no. 910, pp. 833-859, 2008.

[19] K. Petersen, R. Feldt, S. Mujtaba, and M. Mattsson, "Systematic mapping studies in software engineering." in International conference on Evaluation and Assessment in Software Engineering, vol. 8, 2008, pp. 68-77.

[20] G. Guyatt, D. Rennie, M. Meade, and D. Cook, "Users' guides to the medical literature: Essentials of evidence-based clinical practice, (JAMA Archives Journals)," 2008.
[21] I. Inayat, S. S. Salim, S. Marczak, M. Daneva, and S. Shamshirband, "A systematic literature review on agile requirements engineering practices and challenges," Computers in human behavior, vol. 51, pp. 915-929, 2015.

[22] J. Yli-Huumo, D. Ko, S. Choi, S. Park, and K. Smolander, "Where is current research on blockchain technology ? - a systematic review," PloS one, vol. 11, no. 10, 2016.

[23] S. L. Cichosz, M. N. Stausholm, T. Kronborg, P. Vestergaard, and O. Hejlesen, "How to use blockchain for diabetes health care data and access management: an operational concept," Journal of diabetes science and technology, vol. 13, no. 2, pp. 248-253, 2018.

[24] E. Hanna, C. Remuzat, P. Auquier, C. Dussart, and M. Toumi, "Could healthcoin be a revolution in healthcare?" Value in Health, vol. 20, no. 9, 2017.

[25] M. Kang, E. Park, B. H. Cho, and K.-S. Lee, "Recent patient health monitoring platforms incorporating internet of things-enabled smart devices," International neurourology journal, vol. 22, no. Suppl 2, 2018.

[26] A. Engel, "Could blockchain technology add value to surgical outcomes research?" Colorectal Disease, vol. 20, no. 5, pp. 369-370, 2018.

[27] M. Chand, N. Ramachandran, D. Stoyanov, and L. Lovat, "Robotics, artificial intelligence and distributed ledgers in surgery: data is key!" Techniques in Coloproctology, vol. 22, no. 7, pp. 645-648, 2018.

[28] V. Patel, "A framework for secure and decentralized sharing of medical imaging data via blockchain consensus," Health informatics journal, 1460458218769699, 2018.

[29] T. J. Gniadek and P. A. Ball, "Distributed ledgers in transfusion medicine: an opportunity for standards to accelerate innovation." Transfusion, vol. 58, no. $6,2018$.

[30] J. Tung and V. Nambudiri, "Beyond bitcoin: potential applications of blockchain technology in dermatology," British Journal of Dermatology, vol. 179, no. 4, pp. 1013-1014, 2018.

[31] S. H. Lee and C. S. Yang, "Fingernail analysis management system using microscopy sensor and blockchain technology," International Journal of Distributed Sensor Networks, vol. 14, no. 3, 2018.

[32] X. Zheng, A. Vieira, S. L. Marcos, Y. Aladro, and J. Ordieres-Meré, "Activityaware essential tremor evaluation using deep learning method based on acceleration data," Parkinsonism \& related disorders, vol. 58, pp. 17-22, 2018.

[33] A. Dubovitskaya, Z. Xu, S. Ryu, M. Schumacher, and F. Wang, "Secure and trustable electronic medical records sharing using blockchain," in AMIA Annual Symposium Proceedings, vol. 2017. American Medical Informatics Association, 2017.

[34] A. Alsadi, R. Boodoo, J. Taylor, M. Diaz, M. Singh, and T. Patel, "A future for "bitbiopsy" and "cryptospecimen"? proposed use cases of blockchain technology in anatomical and clinical pathology," in Modern Pathology, vol. 31. Nature Publishing Group, 2018, pp. 583-583.

[35] X. Yue, H. Wang, D. Jin, M. Li, and W. Jiang, "Healthcare data gateways: found healthcare intelligence on blockchain with novel privacy risk control," Journal of medical systems, vol. 40, no. 10, 2016.

[36] H. I. News. (2018) The biggest healthcare data breaches of 2018 (so far). https://www.healthcareitnews.com/projects/biggest-healthcare-databreaches-2018-so-far.

[37] W. Gordon, A. Wright, and A. Landman, "Blockchain in health care: Decoding the hype," New England Journal of Medicine Catalyst. Retrieved from http://catalyst. nejm. org/decoding-blockchain-technology-health, 2017.

[38] S. Angraal, H. M. Krumholz, and W. L. Schulz, "Blockchain technology: applications in health care," Circulation: Cardiovascular Quality and Outcomes, vol. 10, no. 9, 2017.

[39] C. Pirtle and J. Ehrenfeld, "Blockchain for healthcare: The next generation of medical records?, DOI: 10.1007/s10916-018-1025-3," 2018.

[40] A. Zhang and X. Lin, "Towards secure and privacy-preserving data sharing in e-health systems via consortium blockchain," Journal of medical systems, vol. 42, no. 8, 2018.

[41] K. N. Griggs, O. Ossipova, C. P. Kohlios, A. N. Baccarini, E. A. Howson, and T. Hayajneh, "Healthcare blockchain system using smart contracts for secure automated remote patient monitoring," Journal of medical systems, vol. 42 , no. 7, 2018.

[42] D. J. Skiba, "The potential of blockchain in education and health care," Nursing education perspectives, vol. 38, no. 4, pp. 220-221, 2017.

[43] H. Li, L. Zhu, M. Shen, F. Gao, X. Tao, and S. Liu, "Blockchain-based data preservation system for medical data," Journal of medical systems, vol. 42, no. $8,2018$.

[44] S. Armstrong, "Bitcoin technology could take a bite out of NHS data problem," BMJ: British Medical Journal (Online), vol. 361, 2018.

[45] Y. Ji, J. Zhang, J. Ma, C. Yang, and X. Yao, "BMPLS: blockchain-based multilevel privacy-preserving location sharing scheme for telecare medical information systems," Journal of medical systems, vol. 42, no. 8, 2018. 
[46] K. Gammon, "Experimenting with blockchain: Can one technology boost both data integrity and patients' pocketbooks?” Nature Medicine, vol. 24, pp. 378-381, 2018.

[47] A. Firdaus, N. B. Anuar, M. F. Ab Razak, I. A. T. Hashem, S. Bachok, and A. K. Sangaiah, "Root exploit detection and features optimization: Mobile device and blockchain based medical data management," Journal of medical systems, vol. 42, no. 6, 2018.

[48] K. Fan, S. Wang, Y. Ren, H. Li, and Y. Yang, "Medblock: Efficient and secure medical data sharing via blockchain," Journal of medical systems, vol. 42, no. 8, 2018.

[49] C. Esposito, A. De Santis, G. Tortora, H. Chang, and K.-K. R. Choo, "Blockchain: A panacea for healthcare cloud-based data security and privacy?” IEEE Cloud Computing, vol. 5, no. 1, pp. 31-37, 2018.

[50] S. Badr, I. Gomaa, and E. Abd-Elrahman, "Multi-tier blockchain framework for iot-ehrs systems,” Procedia Computer Science, vol. 141, pp. 159-166, 2018.

[51] H. Wang and Y. Song, "Secure cloud-based EHR system using attributebased cryptosystem and blockchain," Journal of medical systems, vol. 42, no. 8, 2018

[52] H. F. Atlam, A. Alenezi, M. O. Alassafi, and G. Wills, "Blockchain with internet of things: Benefits, challenges, and future directions," International Journal of Intelligent Systems and Applications, vol. 10, no. 6, pp. 40-48, 2018.

[53] R. Xie, I. Khalil, S. Badsha, and M. Atiquzzaman, "Fast and peer-to-peer vital signal learning system for cloud-based healthcare," Future Generation Computer Systems, vol. 88, pp. 220-233, 2018.

[54] M. A. Salahuddin, A. Al-Fuqaha, M. Guizani, K. Shuaib, and F. Sallabi, "Softwarization of internet of things infrastructure for secure and smart healthcare," arXiv preprint arXiv:1805.11011, 2018.

[55] H. Kaur, M. A. Alam, R. Jameel, A. K. Mourya, and V. Chang, "A proposed solution and future direction for blockchain-based heterogeneous medicare data in cloud environment," Journal of medical systems, vol. 42, no. 8, 2018.

[56] W. J. Gordon and C. Catalini, "Blockchain technology for healthcare: Facilitating the transition to patient-driven interoperability," Computational and structural biotechnology journal, vol. 16, pp. 224-230, 2018.

[57] G. Kyriakoudes, S. Louca, and B. Behbod, "Cyprus's new national health service and future european health,” The Lancet, vol. 392, no. 10157, 2018.

[58] M. N. K. Boulos, J. T. Wilson, and K. A. Clauson, "Geospatial blockchain: promises, challenges, and scenarios in health and healthcare," vol. 17, no. 25, 2018.

[59] Y. Chen, S. Ding, Z. Xu, H. Zheng, and S. Yang, "Blockchain-based medical records secure storage and medical service framework," Journal of medical systems, vol. 43, no. 1, 2018.

[60] P. A. Laplante, M. Kassab, N. L. Laplante, and J. M. Voas, "Building caring healthcare systems in the internet of things," IEEE Systems Journal, vol. 12, no. 3, pp. 3030-3037, 2018.

[61] W. H. Organization et al., "A study on the public health and socioeconomic impact of substandard and falsified medical products. 2017," 2018.

[62] E. A. Blackstone, J. P. Fuhr Jr, and S. Pociask, "The health and economic effects of counterfeit drugs," American health \& drug benefits, vol. 7, no. 4, 2014.

[63] I. BARTON. (2018) Leveraging technology and education in the high-stakes fight against fake medicines. https://www.healthcareglobal.com/pharmaceutical/leveragingtechnology-and-education-high-stakes-fight-against-fake-medicines

[64] F. Clark, "Rise in online pharmacies sees counterfeit drugs go global," The Lancet, vol. 386, no. 10001, pp. 1327-1328, 2015

[65] M. Tremblay, "Medicines counterfeiting is a complex problem: a review of key challenges across the supply chain," Current drug safety, vol. 8, no. 1, pp. 43-55, 2013.

[66] T. K. Mackey, R. Cuomo, C. Guerra, and B. A. Liang, "After counterfeit avastin ${ }^{\circledR}$ - what have we learned and what can be done?" Nature Reviews Clinical Oncology, vol. 12, no. 5, 2015.

[67] J. C. Kohler, T. K. Mackey, and N. Ovtcharenko, "Why the MDGs need good governance in pharmaceutical systems to promote global health," $B M C$ Public Health, vol. 14, no. 1, 2014.

[68] K. Karunamoorthi, "The counterfeit anti-malarial is a crime against humanity: a systematic review of the scientific evidence," Malaria journal, vol. 13, no. 1, 2014.

[69] T. K. Mackey and G. Nayyar, "A review of existing and emerging digita technologies to combat the global trade in fake medicines," Expert opinion on drug safety, vol. 16, no. 5, pp. 587-602, 2017.

[70] P. Sylim, F. Liu, A. Marcelo, and P. Fontelo, "Blockchain technology for detecting falsified and substandard drugs in distribution: Pharmaceutical supply chain intervention,” JMIR research protocols, vol. 7, no. 9, 2018.
[71] J. Fiaidhi, S. Mohammed, and S. Mohammed, "EDI with blockchain as an enabler for extreme automation,” IT Professional, vol. 20, no. 4, pp. 66-72, 2018.

[72] D. G. Glover and J. Hermans, "Improving the traceability of the clinical trial supply chain,” Applied Clinical Trials, vol. 26, no. 11/12, pp. 36-38, 2017.

[73] A. Andrianov and B. Kaganov, "Blockchain in clinical trials-the ultimate data notary," Applied Clinical Trials, vol. 27, no. 7/8, 2018.

[74] M. Gilbert and N. Dasgupta, "Silicon to syringe: Cryptomarkets and disruptive innovation in opioid supply chains," International Journal of Drug Policy, vol. 46, pp. 160-167, 2017.

[75] M. B. Hoy, "An introduction to the blockchain and its implications for libraries and medicine," Medical reference services quarterly, vol. 36, no. 3, pp. 273-279, 2017.

[76] A. Shanley, "Real-time logistics," Real-Time Logistics, vol. 29, no. 10, 2017

[77] E. Funk, J. Riddell, F. Ankel, and D. Cabrera, "Blockchain technology: A data framework to improve validity, trust, and accountability of information exchange in health professions education," Academic Medicine, vol. 93, no. 12, pp. 1791-1794, 2018.

[78] D. Schaffhauser. (2017) Blockchain: Letting students own their credentials. https://campustechnology.com/articles/2017/03/23/blockchainletting-students-own-their-credentials.aspx.

[79] T. Nugent, D. Upton, and M. Cimpoesu, "Improving data transparency in clinical trials using blockchain smart contracts," F1000Research, vol. 5, 2016.

[80] M. L. Anderson, K. Chiswell, E. D. Peterson, A. Tasneem, J. Topping, and R. M. Califf, "Compliance with results reporting at ClinicalTrials.gov," New England Journal of Medicine, vol. 372, no. 11, pp. 1031-1039, 2015.

[81] M. Benchoufi and P. Ravaud, "Blockchain technology for improving clinical research quality,” Trials, vol. 18, no. 1, 2017.

[82] M. Benchoufi, R. Porcher, and P. Ravaud, "Blockchain protocols in clinical trials: Transparency and traceability of consent," F1000Research, vol. 6, 2017.

[83] L. Henderson, “The clinical trial of tomorrow," Applied Clinical Trials, vol. 25 , no. $2 / 3,2017$.

[84] The Coalition Against Insurance Fraud. (2018) By the numbers: fraud statistics. https://www.insurancefraud.org/statistics.htm13.

[85] L. Zhou, L. Wang, and Y. Sun, "MIStore: a blockchain-based medical insurance storage system,” Journal of medical systems, vol. 42, no. 8, 2018.

[86] T.-T. Kuo, H.-E. Kim, and L. Ohno-Machado, "Blockchain distributed ledger technologies for biomedical and health care applications," Journal of the American Medical Informatics Association, vol. 24, no. 6, pp. 1211-1220, 2017.

[87] D. Ivan, "Moving toward a blockchain-based method for the secure storage of patient records," in ONC/NIST Use of Blockchain for Healthcare and Research Workshop. Gaithersburg, Maryland, United States: ONC/NIST, 2016.

[88] L. A. Linn and M. B. Koo, "Blockchain for health data and its potential use in health it and health care related research," in ONC/NIST Use of Blockchain for Healthcare and Research Workshop. Gaithersburg, Maryland, United States: ONC/NIST, 2016.

[89] D. Porter. (2016) Sharing large medical images and files-factors to consider. https://www.getfilecloud.com/blog/2016/12/sharing-large-medicalimages-factors-to-be-considered/.XEJ7oFxKg2x.

[90] C. Brodersen, B. Kalis, C. Leong, E. Mitchell, E. Pupo, A. Truscott, and L. Accenture, "Blockchain: Securing a new health interoperability experience," ed: Accenture LLP, 2016.

[91] A. Azaria, A. Ekblaw, T. Vieira, and A. Lippman, "MedRec: Using blockchain for medical data access and permission management," in Open and Big Data (OBD), International Conference on. IEEE, 2016, pp. 25-30.

[92] A. Fernandez, E. Insfran, and S. Abrahão, "Usability evaluation methods for the web: A systematic mapping study," Information and software Technology, vol. 53, no. 8, pp. 789-817, 2011. 
Mohamad Kassab An associate research professor in Software Engineering at Pennsylvania State University, earned his Ph.D. and M.S. degrees in Software Engineering from Concordia University in Montreal, Canada, B.S. in Computer Science from University of Windsor and B.Eng. in Computer Engineering from the Lebanese American University. Previously, Dr. Kassab has been postdoctoral researcher at ÉTS School of Advanced Technology in Montreal and visiting scholar at Carnegie Mellon University. Kassab's research interests include requirements engineering, system architecture, software quality and measurements, blockchain and The Internet of Things. $\mathrm{He}$ has published extensively in software engineering books and journals. With more than 17 years of industrial experiences, he worked in different industrial roles among which: Business Unit Manager at Soramitsu, Senior Quality Engineer at SAP, Senior Associate at Morgan Stanley, Senior Quality Assurance Specialist at NOKIA and Senior Software Developer at Positron Safety Systems.

Joanna DeFranco An associate professor of software engineering, earned her Ph.D. in computer and information science from New Jersey Institute of Technology, M.S. in computer engineering from Villanova University, and a B.S. in Electrical Engineering and Math from Penn State University. She has worked as an Electronics Engineer for the Navy as well as a Software Engineer at Motorola. Her research interests include software engineering teams, effective teamwork, internet of things, and software intensive critical systems.

Tarek Malas A practicing cardiac surgeon at the Quebec Heart and Lung Institute of the University of Laval. Dr. Malas had previously trained at the Cleveland Clinic Foundation, Harvard University, Emory University Healthcare, McGill University, and the University of Ottawa Heart Institute. His subspecialty interests include valvular heart disease, coronary disease, robotic and minimally invasive surgery, and percutaneous transcatheter therapies. $\mathrm{He}$ also has a passion for research and for addressing public health and healthcare challenges. He completed a Masters in Public Health at Harvard University as well as a Chemical Engineering Degree at McGill University.

Phillip Laplante (M'86-SM'90-F'08) received the B.S., M.Eng., and Ph.D. degrees from the Stevens Institute of Technology, Hoboken, NJ, USA, and an MBA degree from the University of Colorado, Boulder, CO, USA. He is a Professor of software engineering with the Pennsylvania State University, State College, PA, USA. From 2010 to 2016, he led the effort to develop a national licensing exam for software engineers. He has worked in avionics, CAD, and software testing systems. He has authored or co-authored 33 books and more than 200 scholarly papers. His research interests include software testing, requirements engineering, and software quality and management. Dr. Laplante is a Licensed Professional Engineer in the Commonwealth of Pennsylvania and a Certified Software Development Professional.

Giuseppe Destefanis Lecturer in the Department of Computer Science at Brunel University London, with Ph.D. University of Cagliari, MEng and BE, University of Pisa. Previously Dr. Destefanis was Lecturer in the School of Computer Science at the University of Hertfordshire. He has been postdoctoral Researcher at Brunel University and CRIM (Computer Research Institute of Montreal, Canada) and worked closely with the Montreal aerospace industry to support testing activities during the development of flight simulators. While completing his PhD studies, Giuseppe visited the University of Auckland, New Zealand, and the Hong Kong University of Science and Technology. Giuseppe's research areas focus on mining software repositories, empirical software engineering, Agile methodologies, software metrics and patterns, Blockchain and cryptocurrencies.

Valdemar Vicente Graciano Neto An assistant professor with the Informatics Institute at the Federal University of Goiás, Goiânia, Brazil. He received his Ph.D. degree in computer science and computational mathematics from the Institute of Mathematical Sciences and Computation, University of São Paulo (USP), Brazil and the Docteur degree in sciences and information technology from Université Bretagne-Sud (UBS), Lorient, France, in 2018. $\mathrm{He}$ is also the President of the Special Committee on Information Systems of the Brazilian Computer Society (SBC) in the 2018-2019 period. Dr. Graciano Neto is a member of the SBC, the Society for Modeling and Simulation International (SCS) and of ACM. 